\title{
Synchronous Double Malignant Tumors Consisting of Stomach and Hodgkin's Lymphoma with Collision between Gastric Adenocarcinoma and Hodgkin's Lymphoma in the Stomach
}

\author{
Naoki Yanagawa ${ }^{a}$ Shin-ya Ogata ${ }^{a}$ Norimasa Fukushimab \\ Kunihiko Maeda ${ }^{c}$ Gen Tamura ${ }^{c}$ \\ Departments of ${ }^{a}$ Pathology and Laboratory Medicine and ${ }^{b}$ Surgery, \\ Yamagata Prefectural Central Hospital, and 'Department of Nursing, \\ Yamagata Prefectural University of Health Sciences, Yamagata, Japan
}

Key Words

Synchronous · Gastric adenocarcinoma · Hodgkin's lymphoma $\cdot$ Collision

\begin{abstract}
We report the rare case of a 72-year-old man with double cancers (gastric adenocarcinoma and Hodgkin's lymphoma) with collision between gastric adenocarcinoma and Hodgkin's lymphoma. Abdominal computed tomography showed increased wall thickness in the fundus region of the stomach and multiple lymph node swellings in the lesser curvature, periceliac and left cardial regions. Upper gastrointestinal endoscopy showed an ulcer approximately $5 \mathrm{~cm}$ in diameter with a malignant appearance in the fundus region of the stomach. On histopathologic examination, two completely different tumors were recognized in the stomach. One tumor was a poorly differentiated adenocarcinoma characterized by poorly developed tubular structures associated with prominent lymphoid infiltration of the stroma. The other tumor was found to have proliferated in the wall of the stomach, with diffuse granulomatous lesions and bordering the adenocarcinoma. Large atypical lymphoid cells with prominent nucleoli and enlarged mononuclei or multinuclei were seen in the latter tumor. Hodgkin's lymphoma was also found in the swollen lesser curvature lymph nodes. As a result, gastric adenocarcinoma and metastasis of Hodgkin's lymphoma were collided in the stomach. In conclusion, this case might be helpful in exploring the occurrence mechanism of tumor collision between lymphoma and carcinoma.
\end{abstract}




\section{Introduction}

Hodgkin's lymphoma (HL) is a lymphoproliferative disorder that is characterized by Reed-Sternberg cells in lymphoid tissue. The patient's age, gender and general health affect prognosis. HL has a biphasic presentation at the $3 \mathrm{rd}$ and 4 th decades and the 7 th decade. The incidence in males is twice as high as in females. It consists of four subtypes: lymphocyte-rich type, nodular sclerosis type, mixed cellular type and lymphocyte-depleted type. HL is rarely diagnosed with solid tumors [1] and the frequency of HL is low in Japan in comparison with that in Western countries [2, 3]. Gastric cancer is one of the leading causes of cancer-related deaths worldwide, including Japan [4]. The incidence rate of gastric cancer is high in Eastern Asia, and most cases of gastric cancer involve adenocarcinoma. Secondary primary tumors observed in gastric cancer patients have been described by several authors [5-7]. Dinis-Ribeiro et al. [5] reported that the rate of associated second primary malignancy other than gastric cancer was observed to be $3.4 \%$ of patients. Colon cancer constitutes $19 \%$, uterine and ovarian $16 \%$ and breast cancer $13 \%$ of these cases. Eom et al. [6] reported that the incidence of synchronous or metachronous cancer in addition to gastric cancer was $3.4 \%$, and the most common second primary cancer was colorectal cancer, followed by lung and liver cancer. Ikeda et al. [7] examined 2,250 patients with stomach cancer who had colorectal and lung cancer metachronously and renal cell carcinoma synchronously. However, synchronous HL and gastric adenocarcinoma have been rarely reported $[1,8,9]$. In addition, both HL and subtype of gastric adenocarcinoma are thought to be related with Epstein-Barr virus (EBV), but this relation is unclear. Herein, we report the rare case of a 72-year-old man with double cancers (gastric adenocarcinoma with EBV and HL without EBV) with collision between gastric adenocarcinoma and HL in the stomach, and deliberate their relation, particularly the cause of collision.

\section{Case Report}

A 72-year-old man was admitted to Yamagata Prefectural Central Hospital with complaints of epigastric pain and appetite loss. He had no past history or family history of malignancy. Physical examination results were normal and lymphadenopathy was not observed. Laboratory data revealed no significant abnormal findings. Serologic analysis of Helicobacter pylori yielded negative results. Abdominal computed tomography showed increased wall thickness in the fundus region of the stomach (fig. 1a) and multiple lymph node swellings in the lesser curvature, periceliac and left cardial regions (fig. 1b). Splenomegaly was not evident. Upper gastrointestinal endoscopy showed an ulcer approximately $5 \mathrm{~cm}$ in diameter, with a malignant appearance, in the fundus region of the stomach. Therefore, he was diagnosed as gastric cancer.

Total gastrectomy was performed. On macroscopic examination, a type 2 tumor with ulceration was identified in the fundus region of the stomach (fig. 2). On histopathologic examination, two completely different tumors were recognized in the stomach ( $\underline{\text { fig. } 3 \mathrm{a}} \mathrm{a}, \mathrm{b}$ ). One tumor was a poorly differentiated adenocarcinoma characterized by poorly developed tubular structures associated with prominent lymphoid infiltration of the stroma (fig. 3c). The other tumor was found to have proliferated in the wall of the stomach, with diffuse granulomatous lesions and bordering the adenocarcinoma. Large atypical lymphoid cells with prominent nucleoli and enlarged mononuclei or multinuclei (Hodgkin/Reed-Sternberg cells) were seen in the latter tumor (fig. $3 \mathrm{~d}$ ). Immunohistochemical analysis was performed using paraffin-embedded sections and the avidin-biotin peroxidase method. The primary antibodies (clone) used were Bcl-2 (124), CD3 (PS1), CD5 (4C7), CD10 (C8/144B), CD20 (L26), CD30 (1G12), CD79a (JCB117), cytokeratin AE1/AE3 from Nichirei (Tokyo, Japan); and CD15 (C3D-1) from Dako Japan Inc. (Tokyo, Japan). Cytokeratin AE1/AE3 
was only positive in the right upper region. On the other hand, large atypical lymphoid cells were positive for CD15 (fig. 3e) and CD30. These atypical lymphoid cells were also seen in lymph nodes in the lesser curvature, periceliac and left cardiac regions, and this tumor was diagnosed as HL of mixed cellularity. To test for the presence of EBV in our patient, we conducted an EBV-encoded small RNA in situ hybridization test. The HL showed EBV positivity, but the gastric adenocarcinoma did not (fig. 3f). Additionally, H. pylori was not detected in these specimens. Finally, this case was diagnosed as double cancers (HL with metastasis to the stomach and gastric adenocarcinoma).

\section{Discussion}

HL is rarely diagnosed with solid tumors, on the other hand, secondary primary tumors observed in gastric cancer patients have been described by several authors. Synchronous gastric adenocarcinoma and lymphoproliferative disorders have been reported until now, however most of them were combinations with gastric non-HL [10]. Regarding synchronous HL and gastric adenocarcinoma, to our knowledge, only 3 cases have previously been reported in the English literature [1, 8, 9]. Our case appears to be only the fourth reported case of synchronous HL and gastric adenocarcinoma. However, our patient differed from the 3 previously reported cases in that HL metastasized in the stomach and gastric adenocarcinoma were found to collide in the stomach. No such case has previously been reported. Regarding the development of coexisting histogenetically unrelated neoplasms, Tihan and Filippa [11] suggested that these neoplasms may develop due to a genetic predisposition to cancer and/or similar immune-related mechanisms; Tillawi [9] suggested that such a phenomenon may result from two different processes, namely continuous proliferative stimulation by the presence of a common oncogenic factor and the presence of underlying immune abnormalities that pave the way for coexisting malignancies. Recently, the association between synchronous gastric adenocarcinoma and primary gastric lymphoma was found to be related to infection by H. pylori [12]. EBV is also related to some types of lymphoma, nasopharyngeal carcinoma, and certain forms of gastric carcinoma [13]. However, H. pylori was not detected and EBV was only found in HL but not in the gastric adenocarcinoma in our patient. Therefore, we speculate that HL and gastric adenocarcinoma occurred incidentally. Besides, metastasis of HL and gastric adenocarcinoma were collided in the stomach. In this point, our case is very uncommon because most collided gastric adenocarcinomas with lymphomas are combinations of gastric adenocarcinoma with primary gastric lymphoma. As to the mechanism of occurrence of tumor collision, it is considered as follows: (1) continuous chronic stimulation of mucosa by lymphoma cells; (2) immunosuppressive status by lymphoma cells; (3) common oncogenic factor. However, it has been reported that some cancer cells produce chemokine such as lymphocyte migration factor [14, 15]. Gastric adenocarcinoma of our case included many lymphocytes, in other words, adenocarcinoma with lymphoid stroma. Therefore we also hypothesize that gastric adenocarcinoma may produce chemokine and induce HL cells in these cases.

In conclusion, we report the rare case of a 72-year-old man with double cancers (gastric adenocarcinoma and $\mathrm{HL}$ ) with collision between gastric adenocarcinoma and HL. This case might be helpful in exploring the occurrence mechanism of tumor collision between lymphoma and carcinoma. 


\section{Acknowledgement}

We are grateful to Prof. K. Ohshima (Kurume University School of Medicine) and Dr. H. Miyoshi (Kurume University School of Medicine) for their kind advice.

\section{Disclosure Statement}

The authors declare that they have no conflicts of interest.
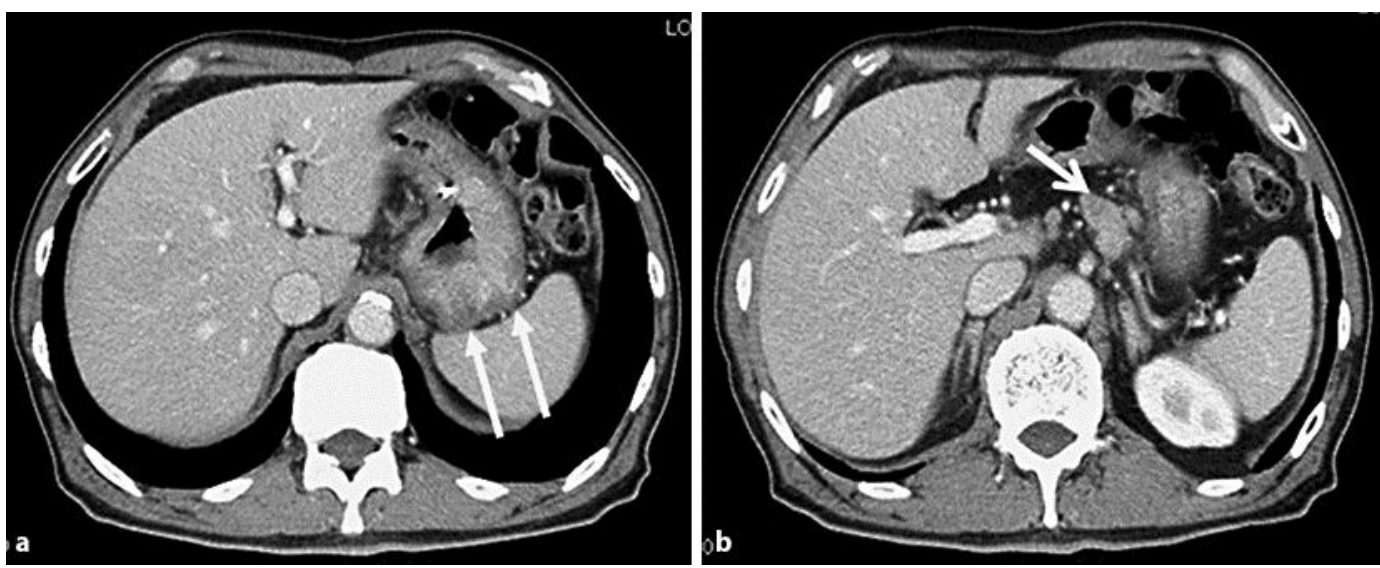

Fig. 1. Abdominal computed tomography showed increased wall thickness in the fundus region of the stomach (a, arrows) and multiple lymph node swellings in the lesser curvature, periceliac and left cardial regions (b, arrow).

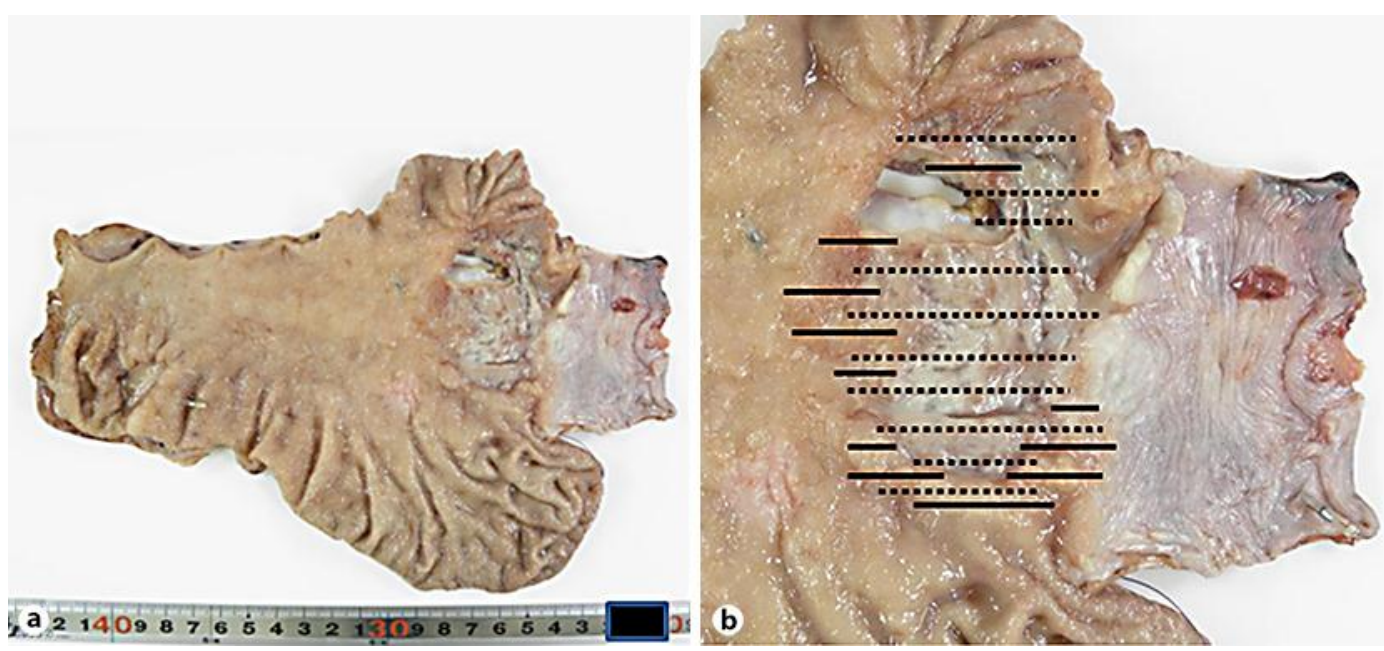

Fig. 2. Macroscopic findings in the stomach. a A Borrmann type 2 tumor was seen in the fundus region of the stomach. b Solid black lines: adenocarcinoma; broken black lines: HL. 

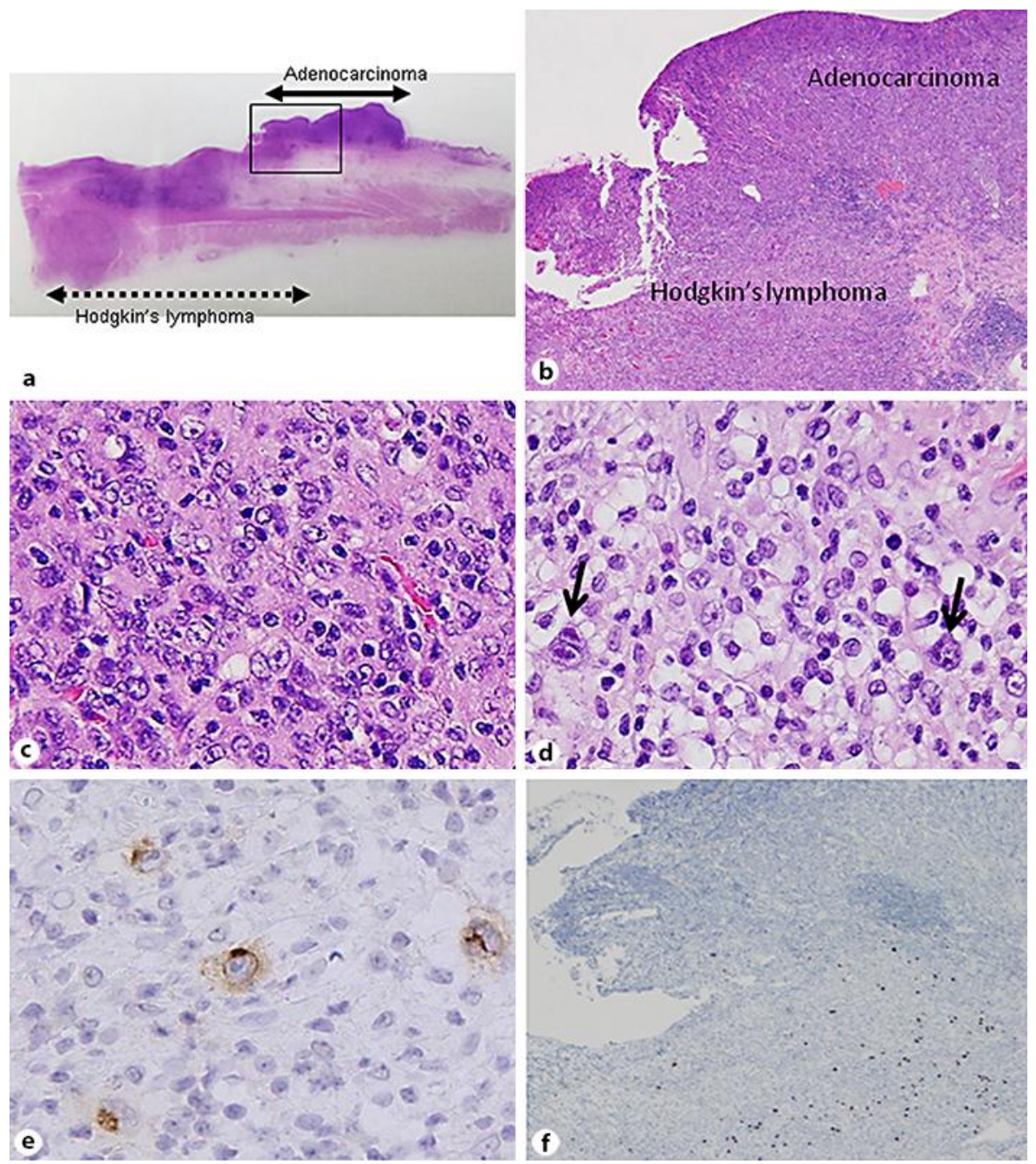

Fig. 3. Microscopic findings. a, b Two completely distinct tumors were identified in the same specimen (right upper: gastric adenocarcinoma; left lower: $H L)$ and collided $(\times 5$ and $\times 40)$. c A poorly differentiated adenocarcinoma characterized by poorly developed tubular structures associated with prominent lymphoid infiltration of the stroma $(\times 200)$. $\mathbf{d}$ Large atypical lymphoid cells with prominent nucleoli and enlarged mononuclei or multinuclei (Hodgkin/Reed-Sternberg cells) were identified (arrows) (×200). e Large atypical lymphoid cells with prominent nucleoli and enlarged mononuclei or multinuclei (Hodgkin/Reed-Sternberg cells) showed CD15 positivity ( $\times 200)$. f EBV was only detected in the left lower region (EBV-encoded small RNA in situ hybridization assay, $\times 40$ ). 


\section{References}

1 Yalçıntaş AU, Öksüzoğlu B, Onder FO, Irkkan C, Üyetürk U, Gökbayrak N, Alkış N: Concomitant Hodgkin's lymphoma and gastric adenocarcinoma: a rare coincidence. Med Oncol 2011;28:251-254.

-2 Lymphoma Study Group of Japanese Pathologists: The World Health Organization classification of malignant lymphoma in Japan: Incidence of recently recognized entities. Pathol Int 2000;50:696-702.

3 Stein H: Hodgkin lymphoma introduction; in Jaffe ES, Harris NL, Stein H (eds): World Health Organization Classification of Tumours, Pathology and Genetics of Tumours of Haematopoietic and Lymphoid Tissues. Lyon, IARC Press, 2001, p 239.

4 Bosman FT, Carneiro F, Hruban RH, Theise ND: WHO Classification of Tumours of the Digestive System. Lyon, IARC Press, 2010.

-5 Dinis-Ribeiro M, Lomba-Viana H, Silva R, Moreira-Dias L, Lomba-Viana R: Associated primary tumors in patients with gastric cancer. J Clin Gastroenterol 2002;34:533-535.

6 Eom BW, Lee HJ, Yoo MW, Cho JJ, Kim WH, Yang HK, Lee KU: Synchronous and metachronous cancers in patients with gastric cancer. J Surg Oncol 2008;98:106-110.

7 Ikeda Y, Saku M, Kawanaka H, Nonaka M, Yoshida K: Features of second primary cancer in patients with gastric cancer. Oncology 2003;65:113-117.

8 Comez G, Pehlivan Y, Kalender ME, Sevinc A, Sari I, Camci C: Synchronous Hodgkin's disease and gastric adenocarcinoma. Oncology 2007;73:422-425.

-9 Tillawi IS: Collision tumor-concurrent involvement of Virchow's lymph node by Hodgkin's disease and metastatic gastric adenocarcinoma. A Troisier's sign and more? Saudi Med J 2007;28:778-782.

10 Hamaloglu E, Topaloglu S, Ozdemir A, Ozenc A: Synchronous and metachronous occurrence of gastric adenocarcinoma and gastric lymphoma: a review of the literature. World J Gastroenterol 2006;12: 3564-3574.

11 Tihan T, Filippa DA: Coexistence of renal cell carcinoma and malignant lymphoma. A causal relationship or coincidental occurrence? Cancer 1996;77:2325-2331.

12 Chan AO, Chu KM, Yuen ST, Leung SY, Lam SK, Wong J: Synchronous gastric adenocarcinoma and mucosa-associated lymphoid tissue lymphoma in association with Helicobacter pylori infection: comparing reported cases between the East and West. Am J Gastroenterol 2001;96:1922-1924.

13 Cohen JI, Bollard CM, Khanna R, Pittaluga S: Current understanding of the role of Epstein-Barr virus in lymphomagenesis and therapeutic approaches to EBV-associated lymphomas. Leuk Lymphoma 2008;49(suppl 1):27-34.

14 Ben-Baruch A: The multifaceted roles of chemokines in malignancy. Cancer Metastasis Rev 2006;25: 357-371.

15 O’Hayre M, Salanga CL, Handel TM, Allen SJ: Chemokines and cancer: migration, intracellular signaling and intercellular communication in the microenvironment. Biochem J 2008;409:635-649. 